\title{
Canadian Academy of Geriatric Psychiatry 2015 Annual Scientific Meeting Abstracts
}

http://dx.doi.org/10.5770/cgj.19.221

\section{Provincial Geriatric Psychiatry Tertiary Mental Health P.I.E.C.E.S. Project}

Michael Wilkins-Ho, Elisabeth Drance

The P.I.E.C.E.S. education program provides the foundation for a vision, language, and approach to the care of older persons with increasingly complex physical and cognitive mental health needs and associated behavioural symptoms. This framework works in concert with the British Columbia BPSD Algorithm and enhances the capacity of the interdisciplinary team to provide care, services and support to those older adults. The BC project started with the Kamloops Integrated P.I.E.C.E.S initiative in 2012 and the Ministry of Health sponsored provincial residential care initiative in 2013.

This project was sponsored by the UBC Tertiary Geriatric Psychiatry Committee working with a grant from the PHSA which brings together representatives from the provincial tertiary centres for Older Adult Mental Health. A provincial project steering subcommittee guided the initiative. Initial education was provided for both educators and leadership, by P.I.E.C.E.S. Canada consultants. This consisted of a 24-hour education workshop to develop 29 P.I.E.C.E.S. educators selected from across the province, who in turn facilitated the 24-hour P.I.E.C.E.S workshops to colleagues. The one-day Leadership Development workshop included representatives from physicians, administrators, and the Ministry of Health. A four-hour P.I.E.C.E.S module for physicians was developed and piloted.

The evaluation is underway using a framework based upon shared solution finding; enhancing and translating knowledge; validating; and acting together/partnering for health-care transformation.

Discussion of the evaluation includes analysis of surveys, narratives, and quality improvement projects; analysis of the physician module; and a summary of resources utilized to date.

Implementation of the First Behavioral Support Team in Acute Care: Lessons Learned after Two Years

Kiran Rabheru, Margaret McKenzie Neil
There are currently Behavioural Support Ontario (BSO)-funded initiatives for persons with dementia and challenging behaviours in long-term care and in the community. In 2013, the Champlain LHIN funded the first pilot BSO team for acute care at the Ottawa Hospital. This initial pilot program is now a fully funded program, and a partnership between Geriatric Psychiatry (GP) and Geriatrics. The supporting strategy that includes ALC LOS, readmission rates of those presenting with dementia, and emergency room aversion.

The BSO was implemented in October 2013. Two registered nurses with specialized training are available by consult. The referring unit is cued to meet the criteria for the consult population through the consult form and must ensure the staff physician is in agreement. This then indicates a medical referral to the GP physician. The BSO nurse expert reviews the case, interviews the patient, designs a care plan, and reviews this with the unit staff and the GP physician. The GP physician will also assess and implement interventions for approximately $90 \%$ of the patients. Follow-up and transitions in care are monitored. An online Recording Log sheet records activity and indicators.

Two years of data support a re-admission rate of $<25$ percent. Transitions in care are enhanced by a referral rate of $25 \%$ of patients to supporting BSO programs post discharge. Case load is increased over 20-25 cases per month. Data and lessons learned from our study for two years will be shared. This program is successful in implementation, increasing a monthly referral rate, and in highlighting a vulnerable dementia population in acute care that is traditionally under-reported.

Can Geriatric Psychiatry Patients Tolerate the Use of Electronic-Based, Self-Report Measures to Help Monitor Their Symptoms?

\section{Ching Yu}

Given the advancement in our information technology, we believe that we can one day make the clinical data from the self-reports readily available to clinicians through the use of electronic devices. There has not yet been a study to determine the tolerability of conducting self-report through electronic devices in older adults (age $>65$ ), who are often 
limited by mobility. The objective of this study is to determine whether, compared to paper self-report psychiatric symptom measures, self-reports using electronic devices (e.g., tablets) are well-tolerated by the patients.

100 geriatric psychiatry outpatients will be studied. The patients will be randomly allocated to taking the questionnaire consisting of self-report measures (BSI-53) in traditional paper-based form vs. on a tablet computer.

We will measure and compare 1) the number of self-report items completed in each group, 2) the length of time required to completing the self-reports, and 3 ) the number of questions each patient asks in order to answer the questionnaire. Furthermore, subjective comments from the patients will be recorded as well. We anticipate comments including, "difficult to read," etc. Additional data, including clinical and demographic data, will also be collected from charts, and analyses will examine the effects of age and other covariates.

We will complete our initial study in the upcoming months. We expect that older adults can tolerate self-measure reports on electronic devices just as well as they would with traditional paper-based reports. The results will help guide us with implementation of information technology in our future practice.

Sahaj Samadhi Meditation May Improve Depressive Symptoms in Late-Life Depression: a Preliminary Analysis of an Ongoing RCT Study

Pramudith Maldeniya, Amanda Arena, Emily Ionson, Amer Burhan, Stephen Wetmore, Ronnie Newman, Akshya Vasudev

Late Life Depression (LLD) has had inadequate treatment response with antidepressants, necessitating additional treatment options. Sahaj Samadhi meditation (SSM), may offer relief for LLD, particularly melancholic symptoms.

Ongoing single-centre, single-blind, longitudinal randomized controlled naturalistic trial to determine if SSM improves mood in patients with LLD $(n=96)$. Patients with LLD are randomized either to SSM plus treatment as usual (TAU) or TAU alone. SSM training, provided by certified teachers, is administered in 120-minute daily sessions on four consecutive days, followed by 60 -minute sessions in each of 11 subsequent weeks. Participants are assessed at baseline and at 4,8 , and 12 weeks.

Preliminary findings from 32 participants who completed the study ( $\mathrm{SSM}=11$; TAU=14) show that SSM led to significant improvement in Hamilton Depression (HAM-D 17) scores compared to TAU alone.

Mean HAM-D 17 scores at baseline were $15.70( \pm 0.86)$ and declined to $12.19( \pm 1.00)$ at Week $12(p<.001)$, whilst TAU group scores did not show a similar decline $(p>.05)$. A subgroup analysis for melancholic depression as assessed by scores on the HAM-D 6 showed a similar significant fall (mean baseline score $=8.19[ \pm 0.47]$ falling to $6.16[ \pm 0.59]$ at Week $12[p<.001)])$ in the SSM arm with no significant change in the TAU arm $(p>.05)$. There was no difference in responsiveness between individuals with early- (age of onset $<50 \mathrm{yrs}$ ) or late-onset ( $>50 \mathrm{yrs}$ ) depression, F (27) = $0.191, p=.665$.

These preliminary findings support SSM as an adjunct treatment for LLD.

\section{Can Early Clinical Exposure to Geriatric Psychiatry Improve Medical Students' Interest in Caring for Older Adults? Protocol for a Future Randomized Controlled Trial}

Soham Rej, Petal Abdool, Chloe Leon, Michael Wilkins-Ho, Paul Blackburn, Karl Looper, Jess Friedland, Vasavan Nair, Tricia Woo, Tarek Rajji

From 2015 to 2031, the number of Canadians aged $\geq 65$ is expected to increase from 5 million to 11 million. Few medical trainees are interested in caring for the elderly, with far less undergoing subspecialized training. We present the protocol for a study testing a medical education intervention to increase trainees' interest in caring for older adults and subspecializing in geriatric psychiatry and geriatric medicine.

Randomized Controlled Trial in four Canadian Teaching Hospitals (at McGill, University of Toronto, and University of British Columbia). Medical students undergoing their 6-8 week third-year clerkship rotation in psychiatry will be randomized to 2-4 weeks of exposure to clinical geriatric psychiatry.

The main outcome will be "interest in caring for older adults as part of my future practice" at the end of their psychiatry clerkship rotation. Secondary outcomes will include 1) comfort working older adults; 2) career interest in subspecializing in geriatric psychiatry; and 3) geriatric medicine.

We will examine bivariate associations between exposure to geriatric psychiatry and interest in caring for older adults, as well as multivariate analyses, controlling for important covariates, (e.g., positive experiences caring for older adults prior to medical school).

We will present the protocol for this upcoming study.

Should our geriatric psychiatry exposure intervention be successful, similar approaches could be widely implemented to help ensure an adequate physician workforce to care for elders, across RCPSC specialties. This could potentially lead to better health-care outcomes for older adults and considerable health system cost-savings.

Education's Impact on Healthy Seniors' Attitudes and Health-Care Preferences Regarding Different Stages of Alzheimer's Disease

Robyn Waxman, Oscar Lu, Barbara Russell 
Alzheimer's disease (AD) and behavioral and psychological symptoms of dementia (BPSD) are not well-known publicly. Studies have yet to explore whether education about both AD and BPSD has an effect on healthy seniors' knowledge, beliefs, and health-care preferences.

As a pilot study, twenty-four female and eight male healthy seniors were quantitatively assessed using AD knowledge, belief questionnaires, and health-care treatment decisional grids at three time-points (pre-, post-, and one-month follow-up) in respect to AD and BPSD educational sessions. Krueger's methodology was used to qualitatively analyze data from focus groups about subjects' reasons for their decisional preferences and any changes.

After receiving education about $\mathrm{AD}$, subjects performed on average $10 \%$ better on the AD knowledge questionnaire. Of the subjects whose knowledge improved overall during the focus group, one-quarter chose less active interventions upon gaining $\mathrm{AD}$ knowledge in the severe stage. One month following, this association strengthened with one-third of the subjects whose knowledge improved choosing fewer active health-care interventions. The majority of both genders chose Alzheimer's disease as a more concerning condition compared to cancer and heart failure. Aggressiveness and psychosis were the most troubling BPSD symptoms for subjects. Medications were the most preferable intervention to manage BPSD symptoms, while physical restraints were least preferable.

This pilot study highlights that education about $\mathrm{AD}$ and BPSD can impact seniors' choices about health-care interventions. This will inform the design of a larger study focusing on seniors with mild cognitive impairment or early $\mathrm{AD}$ where decision-making for care is more time sensitive.

\section{Prevalence of Major Depressive Disorder in Patients with Dementia: a Systematic Review and Meta-Analysis}

\section{Selim Asmer, Dallas Seitz, Julia Kirkham}

There are associations between major depressive disorder (MDD) and dementia. When MDD is present in individuals with dementia it can lead to excess disability and decreased quality of life. While MDD is reported to be higher among individuals with dementia, little is known of the overall prevalence of MDD in persons with dementia and factors that might be associated with an increased prevalence of MDD. In the current study, we completed a systematic review and meta-analysis of the prevalence of MDD in patients diagnosed with Alzheimer's disease and related forms of dementia.

We searched the electronic database MEDLINE from inception until March 2015. We used medical subject headings and free-text words to identify studies that included individuals with dementia diagnosed to standard diagnostic criteria which also reported on the prevalence of MDD according to standard diagnostic criteria. We excluded studies only measuring "depressive symptoms" and those which only diagnosed dementia using only screening tools or MMSE. We used random-effects meta-analysis to determine the overall prevalence of MDD among included studies. Heterogeneity was examined using the Q and I2 statistics. Subgroup analyses to understand factors that may be associated with differences in the reported prevalence of MDD.

We identified 13 studies that met inclusion criteria. These studies were conducted between the years 1994-2010 across in the US $[N=11]$, Canada $[N=1]$, and UK $[N=1]$. Most did not specify the setting from which patients were studied. Prevalence rates ranged from $1.5 \%$ to $43.6 \%$ in the individual studies. A total of 10,286 individuals with dementia were included. In meta-analysis, the overall prevalence of MDD in dementia was $11.1 \%(95 \% \mathrm{CI}: 8.0-15.2 \%, \mathrm{Q}=418, p<.0001$, I $2=95.2 \%$ ). In subgroup analysis, there was no difference in the prevalence of MDD for studies which examined individuals with Alzheimer's disease when compared to vascular dementia (9.5\% vs. $15.5 \%, \mathrm{Q}=1.61, p=.2)$.

MDD is common among older adults with dementia with overall rates that are twice that observed in populations without dementia. However, there are relatively few studies which have examined the prevalence of MDD in populations with dementia, and additional analyses and studies are required to better understand factors associated with differences in the prevalence of MDD in this population.

\section{A Qualitative Assessment of the Use of Entrustable Professional Activities in Psychiatric Residency Training: a Proposed Study}

\section{Robert Madan}

The field of medical education is moving toward using competency-based education. This type of education uses the idea of developmental milestones that are evaluated in a formative manner. Entrustable professional activities (EPAs) is a tool that may be useful in competency-based education. An EPA is a developmental milestone that a trainee must pass to be deemed competent. It is taught and assessed repeatedly over time until the trainee is competent and can be entrusted to do this activity independently. EPAs have yet to be implemented systematically in residency programs and have not been fully investigated.

Our qualitative study will investigate the value, feasibility, use, and barriers to using EPAs in two psychiatry residency subspecialty programs.

Residents in two psychiatry residency subspecialty programs, as well as their supervisors, will be asked to participate in this study. The supervisors and residents will be trained and oriented on how to use this tool. The EPAs will be used at a minimum of every two months and will be formally evaluated at the three- and six-month points in the rotation. After 
completion of two rotations, the supervisors and residents will be involved in focus groups and interviews. Questions will focus on the perceived enablers, barriers, feasibility, use, and value in using EPAs in senior subspecialty residency training.

The focus groups will be transcribed and coded by two research assistants. It is predicted that EPAs will be reported as valuable and that information about the barriers to implementation will be described.

The results of our study will inform Canadian residency programs on how to implement EPAs and move closer toward competency-based education.

\section{The Effect of Weather on Psychiatric Emergency Room Visits and Hospitalization in the Geriatric Population}

Ching Yu

Many patients with severe mental illness are approaching late life. In this population, we expect a high prevalence of frequent psychiatric emergency room visit and hospitalization. Little is known of the impact of seasonal changes, climate, and weather on this population. Our objectives are to identify specific weather conditions or patterns that might affect our geriatric psychiatric patients and predispose them to more frequent psychiatric emergency room visits and hospitalization.

This is a retrospective study of 226 geriatric psychiatric patients admitted to a tertiary-care Canadian inpatient psychiatric unit between 2003 and 2008. We have ascertained their psychiatric diagnoses, their psychosocial parameters (i.e., marital status, living situation).

The main outcomes are psychiatric emergency room visits and hospitalizations in five years following their index psychiatric hospitalization (e.g., 2008-2013 if a patient had been first admitted in 2008).

Our main exposure of interest are daily weather conditions during the same time period (including max. and min. temperatures, precipitation, humidity, sun exposure).

The 226 inpatients' data will be analyzed using bivariate and multivariate analyses (e.g., logistic regression), and the results will be presented at the conference.

We expect that extreme weather conditions would have a negative impact on a specific subgroup of patients (e.g., those living in long-term care facilities), and that extreme weather conditions (e.g., excessive snow and rain) will be associated with less psychiatric emergency room visits and hospitalizations. The association between climate and psychiatric health services, as well as the characteristics of these patients may help guide us to allocate specific resources and provide intervention to prevent emergency room visit and rehospitalization (e.g., home visits, etc) especially during periods of extreme weather conditions.

\section{Attitudes Toward the Behavioural and Psychological Symptoms of Dementia (BPSD) and Implications for Practice}

Robyn Waxman, Oscar Lu, Barbara Russell, Ilan Fischler, Tarek Rajji

Public understanding of dementia typically centers on the problematic symptoms of short-term memory loss and wandering. Other distressing symptoms, known as the behavioural and psychological symptoms of dementia (BPSD), are not well known publicly. They cause significant distress to the person and family, and they constitute one of the major determinants of hospitalization and institutionalization. Lack of knowledge about dementia and BPSD means that care planning involving the patient, family, and clinicians tends to occur too late, which only adds to the patient, caregiver, and system burden.

Decision-guiding powers of attorney are infrequently written, and empirical studies have shown that in their absence substitute decision makers are often inaccurate in choosing treatment options preferred by the patient. Finally, effective care options can be expensive and difficult to access, and pharmacological measures to reduce these symptoms may be associated with serious adverse effects. We will review the results of the pilot study, "Education's impact on healthy seniors' attitudes and health-care preferences regarding different stages of Alzheimer's disease," and we will focus on the subjects' views of BPSD. We will discuss the utility of advance directives with respect to BPSD and learn about clinical pathways being developed for the management of BPSD. Participants were able to consider the effect of BPSD on their health care decisions and state their management choices.

Education and clinical pathways to manage BPSD can help to inform patients when developing their advance directives.

\section{Implementing Guidelines: Integrated Care Pathways and Standards for Agitation and Aggression in Dementia}

Robyn Waxman, Ilan Fischler, Tarek Rajji

Agitation and aggression occur in up to $75 \%$ of patients with dementia. They cause tremendous burden on patients, families, and systems of care. Following a systematic review of the literature and available guidelines and standards for care, the Centre for Addiction and Mental Health (CAMH) and Ontario Shores Centre for Mental Health Sciences (Ontario Shores) have systematically implemented an Integrated Care Pathway (ICP) and a clinical practice guideline, respectively, on their inpatient units. CAMH and Ontario Shores developed different internal processes and outcomes measures with respect to implementing an ICP and guidelines of care, respectively. Consideration was given to deciding on key standards and 
processes, change management, resource allocation, monitoring of clinical outcomes, and onsite expertise. Further, CAMH will discuss an algorithmic approach to ICPs, and Ontario Shores will discuss the role of informatics.

Preliminary results from CAMH and Ontario Shores demonstrate that algorithmic care for agitation and aggression in dementia on an inpatient unit can be successfully implemented. We will also discuss the potential to expand opportunities for greater standardization of care in this area across multiple settings and regions.

The implementation of clinical best practices that are algorithmic in nature and informed by evidence for the assessment and treatment of agitation and aggression in dementia is possible. They may also be associated with reduced unnecessary variability in clinical care, and positive patient outcomes.

\section{Education's Impact on Healthy Seniors' Attitudes and Health-Care Preferences for BPSD}

Oscar Iu

Alzheimer's disease (AD) and behavioral and psychological symptoms of dementia (BPSD) are not well-known publicly, leading to inadequately written advance care directives. However, studies have yet to explore whether education about both AD and BPSD has an effect on healthy seniors' knowledge, beliefs and health-care preferences.

As a pilot study, twenty-four female and eight male healthy seniors were quantitatively assessed using AD knowledge, belief questionnaires and health-care treatment decisional grids at three time-points (pre-, post-, and one-month follow-up) in respect to AD and BPSD educational sessions. Krueger's methodology was used to qualitatively analyze data accumulated using focus groups about subjects' reasons for their decisional preferences and any changes.

Prior to learning about BPSD, for the moderate stage, $63 \%$ of the possible health-care interventions were selected, which decreased to $27 \%$ in the severe stage, as hypothesized. Upon receiving BPSD education, under the condition of moderate-severe dementia, there was a larger portion of health-care options chosen at $35 \%$. More subjects chose hospitalization for tests once they learned about BPSD, which was supported by the Krueger analysis. Aggressiveness and psychosis were the most troubling BPSD symptoms for subjects. Medications were the most preferable intervention to manage their most undesirable BPSD symptoms, while physical restraints were least preferable, followed by seclusion.

This pilot study holds promise in that education about $\mathrm{AD}$ and BPSD can impact seniors' choices about health-care interventions and may be particularly useful for seniors with early signs of dementia. A larger study is warranted.

\section{BPSD and Seniors' Advance Directives}

Barbara Russell

Behavioural and psychological symptoms of dementia (BPSD), especially less well-known symptoms such as disinhibition, cause seniors, family members, and long-term care managers significant ethical concern about using available measures such as "black box" medications, restraints, or seclusion. Moreover public educational materials about advance directives focus primarily on critical care and end-of-life medical interventions.

The study "Education's impact on healthy seniors' attitudes and health-care preferences regarding different stages of Alzheimer's disease" used quantitative knowledge tests and qualitative focus groups. These measures were evaluated for insights relevant to useful, timely advance directives. Also, published medical and bioethics articles about empirical assessments of seniors' advance directives will be presented.

$60 \%$ of study participants had cared for a relative with AD-associated BPSD symptoms. $50 \%$ of study participants had formally appointed a proxy and/or had written instructions. Many participants relied on inaccurate information regarding decision-making processes and effectiveness of and access to critical care interventions. Most participants idiosyncratically prioritized tolerability versus intolerability of high risk medications, restraints, or seclusion for managing BPSD.

Education materials are needed to provide seniors realistic, medically accurate information about benefits-to-harms and effectiveness of common emergency and intensive care measures. Geriatric professionals should advise seniors diagnosed with early $\mathrm{AD}$ about less well-known symptoms so they can decide personally acceptable quality of life and benefitsto-harms trade-offs. These seniors should discuss their preferences with their proxy and, given psycho-emotional burdens on others, family members. Provincially sanctioned substitute decision-making processes and advance directives can be used to respect seniors' preferences when incapable of making such decisions.

\section{Problem Solving Therapy for Older Adults with Depression}

\section{Dallas Seitz, Julia Kirkham, Cindy Grief, Lisa Van Bussel}

Depression is common among older adults, and psychotherapy is frequently recommended as a treatment for depression in this population. Problem Solving Therapy (PST) is a short-term, evidence-based treatment for depression which is being increasingly studied in older adults with depression and other mental health conditions.

This symposium will provide information on the background and history of Problem Solving Therapy along with strategies employed in PST. Information from a recent 
meta-analysis of PST for depression in older adults will be presented in the second session of the symposium. The final presentation of this symposium will present recent experiences with implementation of PST in three geriatric psychiatry programs in Ontario.

The strategies employed in PST are well-suited to the needs of older adults and fit therapeutically with common presentations of depression in this population. Meta-analysis of PST demonstrates that it is a highly effective treatment for depression in older adults with efficacy comparable to other short-term psychotherapies. PST can be adapted and successfully delivered in geriatric psychiatry programs in Canada and PST is acceptable to older adults with depression.

Overall, this symposium will provide attendees with information about PST and practical strategies for incorporating PST in geriatric mental health services.

\section{Implementation and Evaluation of Problem Solving Therapy in Canadian Geriatric Psychiatry Programs}

Dallas Seitz, Cindy Grief, Lisa van Bussel

Background: Problem Solving Therapy (PST) is one of the best supported psychotherapies for depression in older adults. However, to date there is limited information available about the efficacy of PST when implemented in geriatric psychiatry programs in Canada. This presentation will provide an overview of the experiences related to PST implementation in three geriatric psychiatry programs in Ontario.

Methods: Three geriatric psychiatry programs (Queen's University, Western University, Baycrest) have recently trained clinicians in PST. Each program has conducted evaluations related to training, knowledge gained through PST training, and program evaluation as part of program implementation. Facilitators and barriers to program implementation and sustainability have been examined and reviewed at each site.

Results: The total number of clinicians obtaining PST certification has varied between 2 and 14 clinicians to date at the differing sites and the backgrounds of participants have included nursing, social work, and occupational therapy. All sites have implemented PST psychotherapy programs with plans for group psychotherapy at each of the three sites. Patient outcomes measured using the PHQ-9 have indicated that PST is effective in reducing symptoms of depression, and client satisfaction with PST to date has been high.

Conclusions: PST can be implemented in geriatric psychiatry programs in Canada. Preliminary evaluation of PST indicates that it is feasible and acceptable for older adults with major depression with results similar to those observed in other settings. Further evaluation and implementation of PST program may help improve access to psychotherapy and optimize it for specific populations.

\section{Meta-Analysis of Problem Solving Therapy for the Treatment of Major Depressive Disorder in Older Adults}

\section{Julia Kirkham}

Objective: Major depressive disorder (MDD) affects many older adults and is associated with poor medical and mental health outcomes. Problem solving therapy (PST) is emerging as a promising psychotherapy for MDD in older adults although its efficacy of PST in this population has not been well described. We examined the effectiveness of PST for the treatment of MDD in older adults in a systematic review and meta-analysis.

Methods: We searched of electronic databases to identify randomized controlled trials comparing PST to a control condition or other active treatment for the treatment of MDD in adults aged 65 years or older. We used meta-analysis to arrive at pooled summary measures of the efficacy of PST compared to control conditions on the change in depressive symptoms and other outcomes.

Results: Nine studies with a total of 569 participants (290 PST, 279 control) met inclusion criteria. Most studies administered PST in person, and all studies were between 6 and 12 weeks in duration. Meta-analysis of six studies evaluating the effect of PST on depression using the Hamilton Depression Rating Scale (HDRS) identified a significant reduction in depression with PST compared to control conditions (pooled mean difference $=-6.94,95 \%$ CI: -10.91 to $-2.97, d=1.15$, $p=.0006)$. PST was also effective in reducing disability.

Conclusions: The existing research literature on PST suggests that it is an effective treatment for older people with MDD. Further study is required to understand long-term outcomes associated with PST and its efficacy when compared to other treatments.

\section{ECT for Behavioural Symptoms in Major Neurocognitive Disorder}

Lisa McMurray, Daniel Blumberger, Timothy Lau

Behavioural symptoms in major neurocognitive disorder (dementia) are common and distressing to patients and caregivers. Current environmental, psychosocial, and pharmacological treatments have limited efficacy. Emerging evidence suggests that electroconvulsive therapy (ECT) may be effective for treatment-resistant behavioural disturbances. However, its use in this context remains controversial. In addition, many centres have limited experience in using ECT 
with this population. In this workshop, we will summarize the evidence for this modality, explore the controversy surrounding its use, and describe prototypical cases. We will discuss practical issues surrounding the use of ECT in this population and offer suggestions for practice.

\section{Preventing Mood and Anxiety Problems in Older Adults: Two Effective Community-Based Initiatives}

\section{Marie Dussault, Murli Soni}

This interactive workshop will introduce Bounce Back and Living Life to the Full, two innovative evidence-based, low-barrier community programs developed in response to the gap in services for people with depression and anxiety requiring low-intensity interventions. We will highlight a recent Ontario study measuring the impact of delivering Living Life to the Full to older adults, and we illustrate the preventive aspects of these two programs for this often underserved population.

There is limited professional mental health support for the vast numbers of older adults dealing with symptoms of low mood, anxiety and stress - complaints resulting in a high number of visits to primary care. Two programs, Bounce Back and Living Life to the Full, both delivered by the Canadian Mental Health Association, BC Division (CMHA $\mathrm{BC}$ ), are grounded in highly-effective CBT principles. The evidence-based programs follow a brief, structured and solution-focused approach, teaching skills to help identify and change patterns of thinking and behavior causing or maintaining symptoms of distress.

Presenters will briefly describe both programs. Participants will experience a Bounce Back coaching session through a short video clip and will be given the opportunity to participate in a fun, engaging Living Life to the Full class though an exercise reflective of the course content. Final results of an 18-month Living Life to the Full pilot with older adults will be also be shared.

Participants will develop an understanding of both Bounce Back and Living Life to the Full principles and practice as well as their delivery models.

Update on the Role of Non-Pharmacological Treatment Options in the Management of Late-Life Depression and Dementia

Akshya Vasudev, Dallas Seitz, Kimberly Schlegel

There is inadequate treatment response to pharmacological agents used in the fields of late life depression and dementia. Additionally, over the last decade there have been no new or innovative pharmacological agents introduced in the market for either condition.

However, the field of non-pharmacological therapies has expanded with introduction of new treatment options without necessarily the research base expanding at the same pace to guide clinical usage.

This symposium will endeavor to provide a critical review of the available evidence base with various non-pharmacological therapies in the treatment of late-life depression and various dementias. We will also share data from a few clinical trials conducted by the authors.

We shall endeavour to provide direction for further research and service delivery.

\section{Implementing Non-Pharmacological Interventions in Long-Term Care: Results of a Study of Volunteer Visiting Program}

\section{Dallas Seitz}

Background: Non-pharmacological interventions are important person-centred approaches to the management of behavioral symptoms of dementia. One strategy to improve the utilization of non-pharmacological interventions in longterm care (LTC) homes could involve the use of volunteers to provide personalized pleasant activities. Our study investigated the effects of volunteer visits on neuropsychiatric symptoms among LTC residents with dementia.

Methods: We conducted a cluster, randomized controlled trial comparing a volunteer visitor program to usual care. Participants were diagnosed with dementia and had significant symptoms of agitation. Participants received up to three volunteer visits per week over twelve weeks. Volunteers were recruited from community advertisements and were provided with training on dementia prior to volunteering. The primary outcome for the study was the change in the Cohen-Mansfield Agitation Inventory comparing baseline to week 12 and secondary outcomes included the Neuropsychiatric Inventory, Cornell Scale for Depression in Dementia, DemQOL, and Modified Nursing Care Assessment Scale.

Results: A total of 63 participants (16 controls, 47 intervention patients) in five participating LTC homes. Volunteers provided a total of 347 volunteer visits. Each participant with dementia received $336(\mathrm{SD}=192.6)$ minutes and an average of $9.9(\mathrm{SD}=5.6)$ volunteer visits. Overall, individuals in the intervention group had a significant reduction in CMAI total scores during the trial ( $-6.59,95 \%$ CI: -1.07 to $-12.1, p$ $=.02$ ), while individuals in the control group did not have a significant reduction in CMAI total scores $(-4.13,95 \%$ CI: $-13.06-4.79, p=.34)$. However, there was no significant difference between participants in the intervention and control 
groups on their change in the CMAI total score $(-2.45,95 \%$ CI: $-8.12-13.03, p=.63)$. There were also no statistically significant differences noted between the intervention and control groups on the other measures of NPS, depression, quality of life or nursing stress.

Conclusions: We did not observe any significant effects of volunteer visits on the severity of agitation or other neuropsychiatric symptoms over time. We conclude that the volunteer visit program studied in our project is not effective at reducing behavioral symptoms of dementia and that more intensive interventions may be required for this patient population.

\section{Implementing Non-Pharmacological Care Strategies into Practice: Lessons Learned and Future Directions}

\section{Kimberly Schlegel}

Up to $80 \%$ of people with dementia will exhibit behavioural and psychological symptoms of dementia (BPSD). These behaviors may increase suffering for a person with dementia and the burden for their caregivers. BPSD can prompt utilization of more restrictive care options, and might result in the application of both pharmacologic and non-pharmacologic treatments.

Behavioural Supports Ontario (BSO) was initiated as a catalyst for comprehensive system-wide health-care change when supporting older adults with complex behavioural challenges associated with mental health, addictions, or neurological conditions. Within Ontario's South West LHIN, the BSO program has been implemented by embedding nurses and personal support workers with specialized training into longterm care homes. Mobile response teams, who provide urgent, episodic, and transitional support to clients and caregivers, and facilitate knowledge exchange within the health-care system, support further BSO-embedded teams.

BSO has additionally provided funding to adult day programs and Alzheimer's societies. Implementation of this program has not been without its challenges. A review of the literature base, as well as a collection of lived experiences of team members, caregivers, and clients, indicates that caregiver time constraints, our current model of health care, culture of care, and limited educational resources are significant barriers. As BSO enters into the fourth year, quality improvement measures are being put into place to evaluate these barriers and promote success.

\section{Role of Non-Pharmacological Treatment Options in Depression}

Akshya Vasudev
The rate of response to antidepressants is around $40 \%$ with high discontinuation rates due to either lack of response or significant adverse effects necessitating need for other treatment options. Patients are increasingly embracing various non-pharmacological treatments due to perceived health benefits as well as ease of access outside the usual psychiatric practice. Such include yoga and various forms of meditation techniques, which profess to reduce the severity of depressive symptoms. Automatic Self Transcending Meditation (ASTM) is a category of meditation that may be of particular advantage as it is supposed to allow the participant to go quickly into a deep state of relaxation while using a single sound value individualized to the participant.

Results will be shared from an ongoing single-centre, single-blind, longitudinal, randomized, controlled, naturalistic trial to determine if a type of ASTM called Sahaj Samadhi Meditation (SSM) improves mood in patients with LLD (n $=96$ ). Patients with LLD are randomized either to SSM plus treatment as usual (TAU) or TAU alone. SSM training, provided by certified teachers, is administered on four consecutive 120 -minute daily sessions in week one, followed by 60 -minute sessions in each of 11 subsequent weeks. Participants are assessed at baseline, and at 4, 8, and 12 weeks.

Preliminary findings from 32 participants who have so far completed the study will be presented in the symposium.

Additionally the presenter will offer a critical review based on available data of other non-pharmacological strategies in late life depression.

\section{Depression in MCI: a Systematic Review and Meta-Analysis of Prevalence}

Zahinoor Ismail, Heba El Bayoumi, Eric Smith, David Hogan, Corinne Fischer, Tom Schweizer, Scott Patten, Kirsten Fiest

Studies of prevalence of depression in MCI have been inconsistent such that no consensus has emerged. The aim of this study is to systematically review the evidence on prevalence of depression in MCI and generate the best estimate of prevalence and an understanding of heterogeneity in previous studies. MOOSE guidelines were followed and the study was registered with PROSPERO. Databases where searched up to October 2013. References were compiled in Endnote. 9 reviewers participated in the study. Each abstract was reviewed by 2 independent reviewers with the following inclusion criteria for full text review: 1) be original research; 2) report on depression in an MCI population; and 3) be in English. All studies meeting inclusion criteria were then reviewed by a final reviewer (ZI or $\mathrm{KF}$ ) to ensure appropriateness for inclusion in the final analysis. A supplementary search included references of included articles, and an updated database search was done in February 2015. Meta-analysis was performed using STATA. I2 statistics were calculated for the studies and funnel plots were generated. 
5,329 abstracts were reviewed and 209 were selected for full text review. 53 studies were included in the final analysis. Mean study participant number was 398. Mean age of participants was 72.28 . A pooled prevalence of depression in MCI was estimated at $32 \%$, with study heterogeneity based on sample bias and depression instrument used.

Depression is common in MCI. It is important to screen for depression in older adults with cognitive impairment in community and clinical samples.

\section{Pharmacotherapy of Late-Life Bipolar Disorder}

\section{Soham Rej}

Many patients with bipolar disorder are reaching old age. Their pharmacological management is often challenging, especially in light of the medical and cognitive issues that frequently arise in late life. We aim to present some potential guiding principles to facilitate prescribing in late-life bipolar disorder.

We will present data from recent systematic reviews, administrative health-database studies and clinical research studies regarding geriatric bipolar pharmacotherapy.

A limited number of medications have been examined in late-life bipolar disorder, with lithium, valproate, and lamotrigine having the most evidence, albeit mostly from uncontrolled open label studies. In actual clinical practice, psychotropic polypharmacy is highly prevalent $(>85 \%)$, with a high percentage of patients receiving potentially dangerous regimens (e.g., antidepressant monotherapy, multiple atypical antipsychotics). However, medical co-morbidity and medical health utilization may not vary markedly between different medication users (e.g., lithium vs. valproate vs. atypical antipsychotics). Current psychotropic prescribing in Canadian older adults with bipolar disorder may not be as safe and effective as it could be. This may be partly due to fears about medical effects of certain medications (e.g., lithium), which may not be as warranted as previously believed. We present guidance on how to navigate prescribing difficulties in geriatric bipolar disorder.

\section{Meaning-Centered Men's Groups for Men Facing Retirement: a Community Outreach Intervention Designed to Prevent the Onset of Suicide Risk in Later Life}

Marnin Heisel, Gordon Flett, Paul Links, Ross Norman, Sisira Sarma, Sharon Moore, Norm O’Rourke, Rahel Eynan

Older men have the highest rates of suicide worldwide (WHO, 2014). Few intervention studies have investigated suicide risk reduction among middle-aged and older adults, and nearly none has aimed explicitly to reduce risk among men. Men's suicide rates increase at retirement age (Statistics Canada,
2014); retirement may thus be a life transition that can trigger a crisis of meaning, thus increasing suicide risk.

We are recruiting 100-120 community-residing men facing retirement into a multi-stage study to develop, validate, and disseminate Meaning-Centered Men's Groups (MCMG) in community settings. MCMG consists of 12 -session courses of a facilitated group experience for cognitively-intact men, 55 years or older, struggling to transition to retirement, consistent with our research demonstrating a robust negative association between perception of Meaning in Life (MIL) and late-life suicide ideation (Heisel \& Flett, 2014). Group sessions focus on enhancing camaraderie and mutual support, encouraging expression of personal experiences with retirement, and discussing the meaning of work, retirement, leisure, relationships, and generativity. Participants cannot meet criteria for an active untreated mental disorder or participate in concurrent psychotherapy.

We are iteratively evaluating the efficacy of this intervention in preventing the onset or reducing the severity of depression and suicide ideation, and in enhancing perceptions of MIL and satisfaction with retirement, compared with a current-events discussion group control. Groups are being delivered in a community centre, co-facilitated by a community social service worker, to enhance uptake and ensure sustainability. An initial uncontrolled MCMG group is underway with 10 cognitively-intact participants $60-70$ years of age $(M=65.7, S D=3.2)$, of whom 6 are currently retired. Participants reported moderate to strong health ratings and generally positive feelings towards retirement at eligibility assessment, and moderate to strong life satisfaction (Satisfaction with Life Scale; $\mathrm{M}=26.7, \mathrm{SD}=5.3$ ), moderate alcohol consumption (Alcohol Use Disorders Investigation Test; $\mathrm{M}=5.1, \mathrm{SD}=2.4$ ), and low to moderate depressive symptom severity (Geriatric Depression Scale; $\mathrm{M}=7.2$, SD $=6.3$ ) and suicide ideation (Geriatric Suicide Ideation Scale; $\mathrm{M}=45.9, \mathrm{SD}=8.3$ ) at pre-group assessment. Participants have expressed comfort and satisfaction with initial group sessions on a session-by-session feedback form designed to assess their initial experiences with MCMG to help shape this novel intervention. Initial qualitative and quantitative findings will be presented along with lessons learned.

Findings to date support the feasibility of recruiting men concerned about the transition to retirement, into a community-based group intervention. Additional findings will be discussed in the context of the aging population and the need for innovative interventions targeting potentially vulnerable groups.

\section{The Meaning of Stage Congruent Responsive Behaviors (SCRB) in Dementia/Major Neurocognitive Disorders (NCD) and New Classifications Based on Specification of the Theoretical Construct}

Atul Sunny Luthra 
To substantially progress pharmacological and behavioral interventions in dementia and major neurocognitive disorders (NCD), there needs to be an understanding of the meaning of behaviors. The objective of this paper is to identify the limitations of existing literature on assessing and labelling behaviors in dementia/NCD; the biopsychosocial variables contributing to these behaviors; and the specification of theoretical constructs used to classify behaviors into different categories.

Literature was reviewed to identify deficiencies in current dementia/NCD assessments, multifaceted factors contributory to the occurrence of behaviors in dementia/NCD, and a "Specification of the Theoretical Construct" (STC) that justifies aggregation of similar behavioral symptoms into clinically meaningful categories.

Behaviors in dementia/NCD are dichotomized along biological and psychosocial paradigms, and there are significant limitations in the current terminology. Biological (e.g., stage of the disease, inherent circadian rhythms, innate physiological needs), personal (e.g., pre-morbid personality acquired coping mechanisms), and environmental (e.g., milieu structure, interpersonal interactions) factors were identified as playing significant roles in the generation of behaviors in dementia/NCD. STCs identified were theories on information processing; regulation of emotions; compliance and aggression; and motivational and needs-based theories. Twelve behavioral categories were constructed from these theories.

There is complex interplay amongst biopsychosocial factors in the generation of behaviors in dementia/NCD, and a comprehensive model titled Stage Congruent Responsive Behaviors (SCRB) will be used to justify new terminology for these behaviors. This is the first biopsychosocial model for the occurrence of behaviors in dementia/NCD, and this novel classification approach allows for better understanding of the meaning of behaviors.

\section{Positive Psychiatry of Aging in Canada: the Fountain of Health Initiative \& Future Directions}

\section{Keri-Leigh Cassidy, Beverly Cassidy}

With a burgeoning seniors' population and insufficient HR to meet needs, there has been an international call for a "Positive Psychiatry of Aging" for illness prevention and successful aging. The Fountain of Health Initiative is a Canadian project offering new evidence on successful aging and cognitive/ mental illness prevention targeting the public and clinicians. The FoH initiatiative to date has been a Nova Scotia-led initiative over the past five years, developing five key messages disseminated to the public, care providers and patients. Several provincial research projects currently underway will be reviewed, and a new national collaboration through the CCSMH will be discussed.
We found significantly increased clinician knowledge pre- to post- FoH educational sessions and good uptake in using the materials. Feedback on FoH clinical materials by mental health providers, family physicians, and seniors themselves has been positive. Efforts to develop national practice guidelines to promote mental and cognitive health and successful aging are currently underway.

The Fountain of Health Initiative is an important Canadian project offering new evidence about mental and cognitive health promotion to the public and to clinicians. The initiative has formed groundwork for the development of national practice guidelines in this area.

\section{Suicide Prevention Among Older Adults: What Do I Need to Know?}

\section{Marnin Heisel}

Older adults have high rates of suicide in Canada and worldwide, and baby-boomers carry high lifetime rates of suicide. Within the next 20 years, nearly one in four North Americans will be 65 years or older, markedly changing the face of the population and necessitating increased understanding of later life suicide prevention among researchers, practitioners, policy analysts, and advocates.

Older adults often use violent means of self-destruction and are more likely than younger adults to die from their injuries. At-risk older adults often access health-care services in weeks to months prior to death by suicide, creating an opportunity for risk detection and timely intervention. However, clinicians and social service providers typically receive little formal training in working with suicidal older adults. Continuing professional education and knowledge translation efforts are needed to help enhance the care of at-risk individuals; however, not all available information is evidence-based or up to date.

During this interactive workshop, participants will be exposed to late-life suicide prevention knowledge translation resources and will learn about the epidemiology of suicide among older adults, associated risk factors, resiliency processes, and explanatory models, and will gain initial familiarity with approaches to facilitate risk detection and intervention across clinical, community, and population health strata.

Discussion will focus on societal factors that contribute to older adult suicide risk and opportunities for future research.

Medical Morbidity and Mortality after Electroconvulsive Therapy in a Population-Based Sample

Julia Kirkham 
The efficacy of electroconvulsive therapy (ECT) is wellestablished for a number of psychiatric conditions, but information on major medical morbidity and mortality related to ECT is scarce. We examined the incidence of medical adverse events and mortality among individuals receiving ECT and identified predictors of these outcomes.

We undertook a population-level cohort study using administrative health-care databases for patients undergoing a new course of ECT in Ontario between 2003 and 2011. We determined the event rate for all-cause mortality and a composite outcome including 10 specific medical outcomes during and up to 7 and 30 days from the acute ECT course. We used logistic regression to arrive at odds ratios (OR) and $95 \%$ confidence intervals for participant characteristics that were independent risk factors for one or more of these adverse outcomes.

A total of 8,810 unique individuals received an acute course of ECT, corresponding to 135,831 treatments. The all-cause mortality rate was 2.4 per 10,000 treatments at the 7-day end point and 4.8 per 10,000 treatments at the 30-day end point. The rate of adverse medical events was 9.1 per 10,000 treatments and 16.8 per 10,000 treatments for the 7and 30-day end points, respectively. Independent predictors of increased risk for medical morbidity were older age, higher ASA score, history of ischemic heart disease, and longer ECT duration.

Overall, our large, population-based study found that ECT is a relatively safe procedure. Certain patient populations are at higher risk for adverse events following ECT and may benefit from increased surveillance for medical complications.

\section{Delirium and Future Health-Care Use in Older Emergency Department Patients}

\section{Aliya Ramjaun}

Failing to detect delirium in the emergency department (ED) independently predicts mortality within six months after discharge, and up to $76 \%$ of patients exhibiting symptoms of delirium go unrecognized. While risk factors and validated prediction models for delirium have been identified across medical, surgical, and intensive care populations, similar investigations regarding the implications of delirium have yet to be adequately explored in the ED.

A prospective cohort study was conducted, where delirium was assessed as part of an ED contact assessment (ED-CA). Patients were followed-up for 90 days to determine frequency and dates of subsequent ED visits, hospitalizations, and discharge to alternate-level or long-term care (ALC/LTC). Associations between delirium and geriatric syndromes were assessed. A series of univariate logistic regressions were performed to quantify the impact of delirium on experiencing each outcome.
Out of 2,101 patients, $875(42.60 \%)$ were discharged, and of these, $346(39.54 \%)$ returned to the ED. 1,079 (52.53\%) were admitted to hospital at the index ED visit, and 224 (10.91\%) were discharged to ALC/LTC. 287 (14.12\%) patients exhibited delirium symptoms. Nearly all ED-CA items demonstrated significant associations with delirium, with the exception of age, gender, past ED visits, trauma, pain and dyspnea. Delirium was also significantly predicted discharge to ALC/LTC (OR 2.15, 95\% CI: 1.23-3.77) and need for comprehensive geriatric assessment (CGA) (OR 1.73, 95\% CI: $1.11-2.70)$.

A number of associations exist between geriatric syndromes and delirium amongst older ED patients. Delirium may also be predictive of future health-care needs such as ALC/LTC and CGA.

\section{The Relationships between Vascular Risk, Cognition, and Outcome in Late-Life Psychotic Depression}

Kathleen Bingham, Ellen Whyte, Barnett Meyers, Anthony Rothschild, Samprit Banerjee, Alastair Flint

Executive dysfunction and processing speed have been associated with late-life depression (LLD) outcome and with cerebrovascular disease. However, there is mixed evidence directly linking vascular risk to LLD outcome. The aim of our study was to determine whether vascular risk, executive function, and/or processing speed are associated with outcome in late-life psychotic depression. Our hypotheses were that worse baseline executive function and processing speed would be independently associated with poorer treatment outcome; baseline vascular risk would be independently associated with poorer treatment outcome; and vascular risk would mediate the association between the cognition and outcome.

Our study is a secondary analysis of Study of Pharmacotherapy of Psychotic Depression, a 12-week randomized controlled trial. We analyzed data from 142 participants aged 60 years. Independent variables were baseline vascular risk (defined using Framingham Stroke Risk Score [FSRS]), baseline executive function (Stroop colour-word interference score), and baseline processing speed (colour and reading Stroop tasks). Our primary outcome measure was change in depression severity (17-item Hamilton Depression Rating Scale [HDRS]). The data were analyzed with mixed-effects models examining the relation of predictor variables with outcome.

Vascular risk was independently associated with change in HDRS score $(\mathrm{F}=9.91, \mathrm{df}=1,791, p=.0017)$. Neither cognitive measure predicted outcome. Because we did not find a relation between cognition and outcome, we were unable to test for mediation.

Our results support the association between vascular disease and LLD outcome and confirm the use of the FSRS in 
predicting treatment response. We did not find that cognition predicted outcome. Possible explanations will be discussed.

\section{Spaced Retrieval: A User-Friendly Cognitive Rehabilitation Strategy for Persons with Dementia}

\section{Jeff Small}

The presenter has conducted research on and been a consultant for Spaced Retrieval memory training (SRT) since 2009. Research findings indicate that people with dementia can benefit from SRT in the areas of semantic memory (re-establishing face-/object-name associations), prospective memory (remembering to carry out future intended actions), and episodic recent memory (remembering information from a recent event).

In SRT, a client responds repeatedly, over successively longer intervals, to a verbal prompt that is given as a cue to retrieving a piece of information. The success of SRT has been ascribed to its expanding rehearsal schedule in conjunction with a client's relatively preserved procedural memory processing. That is, identification and retrieval of declarative information in SRT is facilitated through implicit and errorless learning. Unlike many other cognitive rehabilitation strategies, SRT requires little cognitive effort and the memory training content is based on its functional utility to the person.

A number of studies have shown that persons with Alzheimer's disease can retain and retrieve information for considerable periods of time following SRT. For these reasons, SRT has the potential to increase a person's confidence and independence in daily life.

The goals of this workshop are to provide participants with opportunities to 1) learn about research evidence supporting SRT, 2) view an app and video of SRT in action, 3) engage in an SRT activity, 4) ask questions and make comments about SRT as it relates to their experience, and 5) take home a clinician-and-family-friendly SRT script.

\section{Considering a Career in Geriatric Psychiatry? Meet the Experts in Education, Research, Advocacy, and Community Practice}

Marla Davidson, David Conn, Kiran Rabheru, Tarek Rajji, Carol Ward, Julia Kirkham, Holly Dornan, Alanna Baillod, Paul Blackburn, Soham Rej

The Canadian Academy of Geriatric Psychiatry (CAGP) Trainee Strategy formed in 2011 with the goal of creating opportunities and support for member-in-training (MIT) CAGP members.

Formal and informal feedback has shown that trainees often wonder "What is a geriatric psychiatrist?" and "What would a career in geriatric psychiatry entail?" Trainees have indicated that they are interested in learning more about the lifestyle and career of a geriatric psychiatrist from experts in the field.

In response to trainees' feedback, an interactive workshop has been created by the CAGP Trainee Strategy to explore career opportunities within geriatric psychiatry. This workshop will involve a brief introduction of the topic by co-chairs Dr. Marla Davidson and Dr. Soham Rej followed by a 30-minute panel presentation by experts in the field of geriatric psychiatry.

Dr. David Conn, Dr. Kiran Rabheru, Dr. Tarek Rajji, and Dr. Carol Ward will describe why they chose a career in geriatric psychiatry, while highlighting the various opportunities they have had during their careers and sharing their perspective of the lifestyle of a geriatric psychiatrist.

The second half of the workshop will involve trainees interacting within small groups in focused discussions with the panel experts in the areas of education, research, advocacy and community practice. This is a great opportunity for residents, fellows, and students from all disciplines to network with experts and colleagues and to ask questions about career development in a friendly setting.

\section{Innovations in Health Care Delivery: Recognition and Management of Behavioural Disturbances in Elderly Patients Admitted Into Acute Care Hospital Settings}

\section{Kiran Rabheru, Lesley Wiesenfeld, Dallas Seitz}

Acute medical or surgical care is often necessary for older patients with cognitive impairment. In addition to their complex medical or surgical needs, a large number of them also demonstrate responsive behaviors, a well-known predictor of prolonged length of stay (LOS), alternate-level care (ALC), and gridlock in the Emergency Room (ER). In January 2015, over 4,000 ALC patients occupied $14 \%$ of acute and postacute-care beds in Ontario hospitals, largely due to their complex medical and behavioural health-care needs. An innovative, system-wide approach is critical to target these barriers to discharge, in order to alleviate health-system pressures and improve access to acute care.

To this end, the Behavioural Supports Ontario (BSO) Program, primarily focused on seniors living within the community and long-term care settings, has had a modest positive impact in terms of behavioural management. However, the care of patients within acute-care hospitals has largely been neglected and presents an opportunity for further enhancement of care, individually and system-wide.

This symposium will present the work of three acute-care hospitals in Ontario focusing on the needs and challenges of behaviourally disturbed cognitively impaired elderly patients that they serve. 
1. Dr. Kiran Rabheru (Ottawa Hospital, Ottawa, Ontario) will present findings from the first provincially funded BSO program in Ontario within a large, in-patient, tertiary-, acute-care hospital.

2. Dr. Lesley Wiesenfeld (Mt. Sinai Hospital, Toronto, Ontario) will present findings from an acute-, tertiary-care, in-patient hospital setting.

3. Dr. Dallas Seitz (Providence Care, Queens University, Kingston, Ontario) will present findings from the perspective of his team that supports and assists the care of patients within several community acute-care hospitals

It is critical to understand the unique features of dementia care within acute-care settings and the importance of collaborating with community partners for a smooth transition with discharge planning. Data to support a systematic approach to this and to improve the care of this population will be presented.

It is critical to recognize the important role played by geriatric psychiatry and enhanced behavioural support teams within acute-care settings. Collaborating with medical, surgical, psychiatric, and Emergency Room colleagues is essential for optimal care and outcomes.

\section{Essential Skills for the Geriatric Psychiatrist in the Era of Competency-Based Education (CBE)}

Karen Saperson, Melissa Andrew, Mark Bosma, Sheila Harms

The Future of Medical Education in Canada project describes a need for a paradigm shift in medical education which moves away from the current time-based model to a framework which defines, teaches, and evaluates learner competency across the continuum. The Royal College is implementing the CBD (Competence by Design) initiative within the CanMEDs 2015 Framework for residency programs over the next few years.

The faculty development efforts required for successful implementation of this model has been referred to as the "Achilles Heel" of CBE, given that dedicated faculty with established patterns of teaching, will be required to fundamentally change patterns of imparting knowledge and assessing learners.

In this workshop, we will define terminology used in CBE and demonstrate how key concepts might be applied in the clinical context of geriatric psychiatry. We will invite audience participation in discussing challenges inherent in improving supervisor knowledge and skills, for the purpose of providing meaningful feedback to enhance resident competencies. Furthermore, we will propose a framework for promoting faculty development initiatives.

The success of the Royal College CBD initiative resides largely within the ability to engage faculty in enhancing their ability to teach and evaluate resident skills within this model.

\section{Benzos and Beyond: Emerging Trends in Prescription Drug Abuse}

\section{Aviva Rostas, Uri Wolf}

Abuse of prescription drugs constitutes a growing problem in the geriatric population. Numerous risk factors exist for prescription drug abuse in older adults including social isolation, polypharmacy, poor health status, and previous or current substance use disorder. Literature on this under-recognized clinical problem in the elderly has not focused on several of the antidepressants, antipsychotics, and other psychotropic medications which have only recently been recognized in the literature as having abuse potential. In particular, recreational use of bupropion via oral, nasal, and intravenous routes has been documented as a rising concern in non-geriatric populations.

Here, we describe a case of a geriatric patient with persistent depressive disorder and a history of substance use who developed hypomanic symptoms and hypertension in the context of bupropion abuse. The association between older age and prescription drug abuse is explored, and the literature with respect to three prescription medications with lesser-known abuse potential (bupropion, quetiapine, and tricyclic antidepressants) is reviewed.

We identify an approach to the assessment and management of prescription drug abuse in older adults, with a demonstration of motivational interviewing techniques. In this instance, the patient ultimately terminated the therapeutic relationship when further prescriptions for bupropion were not provided.

This case adds to an emerging literature documenting the abuse potential of several commonly prescribed antidepressants, antipsychotics, and other psychotropic drugs. We propose that health-care providers serving the geriatric population exercise additional caution when prescribing these medications to patients with risk factors for substance abuse.

\section{Treatment-Resistant Depression and Generalized Anxiety Responding Solely to Pregabalin and Ketamine: Is There a Sub-Syndrome Responding Only to Drugs Modulating Glutamate Neurotransmission?}

\section{Simon Davies}

A 61-year-old man with a four-year history of severe depression was socially isolated and unable to work. At CAMH, he had utilized numerous treatments including multiple antidepressants (SSRIs, SNRIs, bupropion, trazodone, and nortriptyline), antipsychotics (quetiapine, aripiprazole), methylphenidate, hydroxyzine, testosterone patches, ECT, 
and rTMS. Most were not tolerated; the remainder were ineffective. On further consultation, he had both severe depression (BDI 37/63) and marked symptoms of generalized anxiety disorder (GADI 50/72) and was taking clonazepam and zopiclone.

He was prescribed pregabalin (licensed in many countries for GAD). Although tolerating only $125 \mathrm{mg} /$ day, he responded well, resuming many activities abandoned during his prolonged illness. He successfully reduced clonazepam and zopiclone intake. BDI score initially improved to $19 / 63$ and briefly to $8 / 63$ (remission), stabilizing in the mild range (14-19/63) over 18 months. GADI score initially improved to $30 / 72$, slipping back gradually to $50 / 72$.

On referral to Toronto Western Hospital, ketamine was prescribed for intranasal inhalation $100-125 \mathrm{mg} q 3$ days. At seven months, his depression was in remission (BDI 12/63). His GADI score improved to 40/72. He continued to expand his repertoire of social activities and could work some weekdays for his brother's business - an impossibility over the previous five to six years.

Having failed on multiple pharmacological and physical treatments, he improved substantially on pregabalin and ketamine. Both drugs modulate glutamate neurotransmission. This suggests the existence of a resistant depression/anxiety syndrome responding only to treatments influencing glutamate, and raises the possibility that response to pregabalin could be a predictor of positive response to ketamine.

\section{The "Old" Woman}

Shabbir Amanullah

An 82-year-old woman with declining memory presented with agitation but also an odd belief around her age.

People of all ages hold aging-related attitudes. Even very young children recognize age differences and evidence attitudes that appear to be generation specific. Since attitudes presumably affect behaviour and since long life has recently become the rule rather than the exception, interaction with old persons and personal outlooks toward growing old may well be influenced by one's general aging-related beliefs. Attitudes of younger persons and concepts pertaining to an overvaluation of aging; defining the differences in normative aging versus a pathologic process is influenced by the availability, accessibility, adequacy, and acceptability of services intended for use for older persons.

We reviewed the literature and looked at existing evidence for the different aspects of ageing, specifically focusing on attitudes, beliefs, and stigma. Pubmed, CINHAL and conference proceedings were reviewed.

Ageing is a complex process with significant variations across cultures and more importantly some religious groups. The impact of ageing is even more significant in the western context where an inordinate importance is placed on youthfulness. This forces in many ways older people to develop unique coping mechanisms.

The process of ageing is still poorly understood from the biological point of view but even more poorly understood from the phenomenological point of view. This needs much work. 